\title{
Exploring perceptions of alcohol use in two Indian states: A qualitative study from Delhi and Haryana, India
}

\author{
Monika Arora ${ }^{1,3}$, Abha Tewari ${ }^{1}$, Shalini Bassi ${ }^{1,3}$, Kavita Chauhan ${ }^{1}$, Shikha Bhasin ${ }^{1}$, Ann-Sofie Bakshi ${ }^{2}$, and Sven \\ Andréasson ${ }^{2}$ \\ ${ }^{1}$ Public Health Foundation of India, Haryana, India \\ ${ }^{2}$ Karolinska Institutet and Stockholm Health Care Services, Centre for Psychiatry Research, Department of Clinical Neuroscience, Stockholm, \\ Sweden \\ ${ }^{3}$ Health Related Information Dissemination Amongst Youth, New Delhi, India
}

\begin{abstract}
Aims: To explore perceptions and determinants influencing alcohol consumption, with a view to inform development of suitable policies to control alcohol consumption.

Methodology: Focus group discussions (FGDs) $(n=24)$ were conducted in two Indian states among the rural Haryana ( $n=8$ FGDs; 58 participants) and urban Delhi ( $n=16$ FGDs; 113 participants) populations. Purposive sampling was used to recruit the participants, who were from high and low socioeconomic status (SES) groups and in the age group of 18-58 years.

Results: Different factors influence alcohol consumption in high and low SES groups. The age of initiation is now as low as 10-12 years, attributable to the recent trend of increased accessibility. The participants agreed that alcohol consumption has now become a more acceptable part of daily living, whereas earlier it was limited to special occasions and festivals. This has contributed to an increase in drinking. Drinking among women was earlier a taboo, but now women (mainly of high SES), are often seen drinking at social gatherings. The respondents mentioned weak enforcement of regulatory policies related to alcohol consumption and sales.
\end{abstract}

Conclusions: A shift in attitudes was observed towards drinking. Drinking is increasingly seen as a part of daily life. The availability of alcohol is perceived to be increasing and restrictions on sale are not effectively enforced. There is an urgent need to re-evaluate the alcohol policy from a public health perspective in India.

\section{Introduction}

Though patterns of alcohol consumption vary widely across the world, it is the third-largest risk factor for disease and disability, particularly in middle-income countries (World Health Organization, 2011). The per capita consumption of alcohol in India has increased rapidly and is estimated to be 4.3 liters (Sassi, 2015). A disturbing development is the initiation of drinking at younger ages (Gururaj, Murthy, Rao, \& Benegal, 2011). In India, alcohol is consumed individually, socially, ritualistically, and to get drunk (Bennett, Campillo, Chandrashekar, \& Gureje, 1998). India has seen a rapid proliferation of city bars and nightclubs in recent years and is "fast shedding its inhibitions about alcohol as a lifestyle choice” (Lancet, 2009). Changing norms, along with a rise in disposable income, have led to an increase in drinking behavior. In Haryana, the green revolution brought prosperity, leading to an increase in alcohol consumption, giving rise to alcoholism and wife-beating (Maheshwari, 2004). Although alcohol use by women is socially stigmatized in rural India, there is an increase in alcohol consumption among women from urban settings (Gururaj et al., 2011). Tribal areas have the highest prevalence of alcohol use both among men and women (Benegal, Gururaj, \& Murthy, 2003). Caste is significantly associated with the consumption of alcohol. Men from the Scheduled Castes and Scheduled Tribes, the most disadvantaged socioeconomic groups in India, are 1.4 times more likely to use alcohol than others (National Sample Survey Organization, 2005).

Alcohol is a state subject in India, and most state policies are formulated to earn revenue from alcohol products. The minimum legal drinking age in Delhi and Haryana is 25 years, but it is 21 years in the neighboring state of Uttar Pradesh. A unique system of regulation exists, where alcohol is not sold on certain days, which are designated as

Correspondence: Dr. Monika Arora, Director-Health Promotion Division and Associate Professor, Public Health Foundation of India, Plot No. 47, Sector-44, Institutional Area, Gurgaon 122002, Haryana, India, Telephone: 0124-4781400; Email: monika.arora@phfi.org

Financial support: The research was funded by a grant from the Swedish International Development Cooperation Agency [Grant number: 2111051]

Declaration of interest: We declare that we do not have any competing interests.

Keywords: Alcohol, Perceptions, Determinants, Alcohol Control Policies, Qualitative 
"dry days" and usually fall on major religious festivals or national holidays. There is a ban on advertising of alcohol products, their sale being controlled through state excise policies. To counteract this, alcohol companies use surrogate advertising and engage with the social media in different ways to promote their brands. Surrogate advertising is used to promote music CDs, packaged drinking water, and other items under the same brand name as the alcohol product (Public Health Foundation of India, 2013). Research has established an association between the depiction of alcohol drinking in Indian movies and increase in alcohol consumption among younger population (Nazar, Arora, Gupta, Singh, \& Sargent, 2012).

Increased exposure through extensive and enticing alcohol advertisements, increased availability through more outlets, and relaxation of overseas trade rules (Esser \& Jernigan, 2015) have contributed to a rise in use of alcohol. With increasing urbanization and industrialization, people in India have become exposed to Western culture, where alcohol use is socially and culturally acceptable.

A knowledge of the people's perceptions of alcohol use and a contextual understanding of the determinants influencing their environment will aid in tailoring interventions to target these determinants from the policy and community outreach perspective. This study aimed to explore people's perceptions of alcohol consumption in India to inform the development of high-impact alcohol control policies.

\section{Methods}

Twenty-four focus group discussions (FGDs) were conducted with participants from rural and urban areas in two states: Delhi ( $n=16$ FGDs; 113$)$ and Haryana $(n=8$ FGDs; 58 ). The participants were in the age range of 18 58 years, divided into two groups: $18-37$ years and 38-58 years. Separate FGDs were conducted with male and female participants (Table 1). The participants were recruited from three groups: urban high socioeconomic status (SES), urban low SES, and rural. The homogeneity of the groups was ensured on the basis of age and gender (in both rural and urban areas) and SES (in urban areas only). As questions related to alcohol use were deemed sensitive, the FGDs were not based on the participants' alcohol consumption status. The study was undertaken between January and December 2013.

\section{Study participants and data collection}

A purposive sampling approach was adopted to recruit participants with the help of a local non-government organization and residents' welfare associations. Informed consent was obtained from the participants before their recruitment for the FGDs, and they were assured confidentiality of their responses. Ethics approval was obtained from the Institutional Ethics committee at the Public Health Foundation of India. Overall, four moderators and four note-takers collected the data. Each FGD was conducted by one moderator and one note-taker. These team members were trained in following study protocol and using FGD guides as well as in documentation of collected data. Each group had 7-8 participants, both users and non-users of alcohol. All FGDs were held within the community and lasted 45-60 minutes. The proceedings were audio-recorded, transcribed, and translated into English from Hindi (the local language).

\section{FGD questions}

An FGD guide was developed based on review of the literature and research questions. The FGD guide (Table 2), consisting of semi-structured questions in English, was translated into Hindi. Themes were identified to help direct the discussions, although the participants were allowed to independently give their own opinions, with minimal intervention from the researcher (Farooqi, Nagra, Edgar, \& Khunti, 2000). The questions aimed to elicit responses on the type and quantity of alcohol consumed, the frequency of alcohol use, attitudes towards drinking, expenditure on alcohol, reasons for drinking, sources of purchase of alcohol, and how access affects alcohol consumption

Table 1

\section{Details of FGD participants}

\begin{tabular}{|c|c|c|c|}
\hline \multicolumn{2}{|l|}{ Urban } & \multicolumn{2}{|l|}{ Rural } \\
\hline \multicolumn{2}{|c|}{4 FGDs with high socio-economic status (SES) males in urban area } & \multicolumn{2}{|c|}{4 FGDs with males from rural area } \\
\hline 0 & 2 FGD aged $18-37$ yrs & 0 & 2 FGD aged 18-37 yrs \\
\hline 0 & 2 FGD aged 38-58 yrs & 0 & 2 FGD aged 38-58 yrs \\
\hline \multicolumn{2}{|c|}{4 FGDs with low SES males in urban area } & \multicolumn{2}{|c|}{4 FGDs with females in rural area } \\
\hline 0 & 2 FGD aged $18-37$ yrs & 0 & 2 FGD aged $18-37$ yrs \\
\hline 0 & 2 FGD aged $38-58$ yrs & 0 & 2 FGD aged $38-58$ yrs \\
\hline \multicolumn{4}{|c|}{4 FGDs with high SES females in urban area } \\
\hline 0 & 2 FGD aged $18-37$ yrs & & \\
\hline 0 & 2 FGD aged 38-58 yrs & & \\
\hline \multicolumn{4}{|c|}{4 FGDs with low SES females in urban area } \\
\hline 0 & 2 FGD aged $18-37$ yrs & & \\
\hline 0 & 2 FGD aged 38-58 yrs & & \\
\hline
\end{tabular}


.Table 2

FGD Guide

\begin{aligned} \hline S. No. & Questions \\ \hline 1. & $\begin{array}{l}\text { In your community/neighborhood what kind of alcohol do } \\ \text { people drink? Are there any special groups of people that } \\ \text { drink alcohol (age, social class, gender, etc.)? }\end{array}\end{aligned}$

2. What are the attitudes in general towards drinking alcohol in your community/neighborhood?

3. In your opinion what are the various reasons for which people consume alcohol?

4. How do people access alcohol beverages? From where do they get their drinks and what are these places? Do you think it is easier or more difficult to get a drink today as compared to 5 years back or 10 years back?

5. What are the things that people do (behavior and activities) after they consume alcohol? If so, what are they?

6. In your opinion when does use of alcohol turn into misuse?

7. Have you seen any alcohol-related problems in your neighborhood community? What kind of problems?

\section{Probes}

Where do they buy alcohol? What is the average cost of the drink?

How much and how often do they drink?

Where do they drink? At what time do they drink?

When do they drink/on what occasions?

Are there different attitudes on drinking concerning different groups: is it more acceptable for some people/groups to consume alcohol, more unacceptable or shameful for others?

When is it acceptable to consume alcohol? When is it unacceptable?

Probe for both positive and negative reasons, like for fashion, peer pressure, fun, due to stress, youth and women drinking, experimentation, initiation and continued drinking; age of initiation

Probe: sources such as friends/relatives; shop purchase/ stealing/borrowing; free supply for profit or advertising

Probe for both positive and negative consequences: for fun, relaxing, socializing, long term and short term consequences; for the alcoholic, for his family/spouse/children, for the society as a whole; social/financial/health

Any kind of crime against women and children, other crimes; harm to others/self, e.g., social and physical; environmental and infrastructure damage, nuisance; indulgence in other substance abuse

\section{Data Analysis}

Each transcript was read several times, and the data were coded and organized under themes and analyzed. NVivo software was used to develop the coding scheme. Five major themes were generated, as explained below.

\section{Results}

\section{Change in social norms related to alcohol use}

All participants felt that the use of alcohol has become very common. The pattern of alcohol consumption has changed from occasional and ritualistic use to one of social use: "Today, children and their fathers drink together. Sometimes parents sit together and drink" (female, high SES, 18-38 years).

The majority reported that while a decade ago, only men used to drink at social gatherings, today women are often seen drinking on such occasions. However, this change in trend is noted mainly among women of high SES groups. Participants from rural settings said that the culture has changed only in urban settings, where women and young girls are more open to drinking. Women rarely drink in the villages. Young children, especially boys, have begun drinking more frequently.

\section{Consequences of alcohol consumption}

Most participants were aware of the harmful effects of drinking on health and stated that consuming alcohol causes various diseases such as liver cirrhosis, liver problems, and cancer, and might eventually lead to death.

The participants were of the opinion that alcohol consumption does not economically affect the middle- and higher-income groups. The lower-income groups, especially those with meager earnings, were thought to be the worst affected by alcohol consumption: "People here are already poor. When a person gets addicted to alcohol, the financial condition of the family gets even worse" (male, low SES, 18-38 years).

While discussing the consequences of alcohol use, the female participants emphasized social issues such as domestic violence and rape, while the male participants focused on road accidents, thefts, and other crimes. Female participants from the rural areas narrated their experiences in their households and mentioned that those addicted to alcohol are often violent and aggressive. The amount spent on alcohol is at times higher than the family's monthly income. The intense urge to drink may even force people to sell their household items, leading to domestic violence and ultimately affecting the community: "People in our neighborhood are often found selling bed sheets and lentils ... they even sell the kneaded dough to get money and drink alcohol” (female, rural, 38-58 years). 
Female participants from high SES said that if a person in the family is alcoholic, the matter is mostly kept confidential. He is then married off in the belief that he will quit drinking after marriage. However, nothing changes and his wife and children suffer, and ultimately, the grandparents take care of the entire family.

\section{Trends in alcohol use}

The majority of participants stated that people who drink usually consume all kinds of alcohol, from expensive branded liquor to less expensive. People from the middleand higher-income groups prefer expensive brands of hard liquor (Indian-made foreign liquor), beer, and wine. People of low SES usually consume cheap, low-quality, and locally prepared country liquor: "People from rural areas drink cheap-quality alcohol and people from urban areas drink expensive alcohol. Servants and ragpickers buy alcohol worth 15 rupees, sold in packets, which is very cheap and often adulterated” (female, high SES, 38-58 years).

Women usually consume white drinks (vodka and rum), and men prefer whisky and beer: "Normally what I have seen is that ladies prefer consuming white spirits, whereas men consume brown liquors" (male, high SES, 38-58 years).

Females of low SES hardly consume alcohol: "In our area, there are only one or two women who drink alcohol, while men mostly drink daily and heavily" (female, low SES, 1838 years).

Male participants of high SES said that their family and friends usually drink a little $(30 \mathrm{ml})$ in one sitting. Male participants of low SES said their friends consume anywhere from one quarter to a full bottle or two. This difference between the alcohol consumption of males of high and low SES was attributed to the acceptance of such behavior in their respective communities. Drinking heavily and getting drunk is socially acceptable, to some extent, to those of low SES, whereas being presentable at all times is a must for those of high SES: "They drink so much in the night that the effect is there till the next morning" (female, low SES, 18-38 years). Heavy and frequent drinking was also commented upon by rural participants: "They [alcohol users from rural settings] just keep on drinking from morning till evening” (male, rural, 18-38 years).

The majority of participants reported that people usually consume alcohol at home. However, those who drink heavily drink wherever they find company and a place to sit (parks, streets, outside liquor shops, cars, under trees, and while working in the fields). People of higher SES drink at home and at social gatherings (bars, restaurants, etc.), whereas those of low SES may be found drinking on the streets, in parks or other such public places, at home, and at social gatherings.

Almost all participants stated that people of all ages consume alcohol nowadays. The age of initiation of alcohol use has decreased drastically over the past decade; even school children of the age of 10-12 years have started drinking. Children usually start consuming alcohol out of curiosity and to replicate the habits of their elders: "Children in all age groups are drinking these days . . . 10year, 15-year, 20-year-olds are drinking” (male, low SES, $38-58$ years).

The participants stated that alcohol consumption increases during festivals, marriages, and social gatherings, and some people see such occasions as an excuse to drink: "On Holi, Diwali, Dussehra [major Indian festivals], and during marriages, people tend to drink more. Festivals are just excuses to drink" (male, low SES, 18-38 years).

Alcohol consumption also increases during election time, as alcohol is distributed by various political parties (which is illegal) to garner votes in their favor: "During elections, a lot of alcohol is distributed by the political parties. People go and get drinks from every party and drink heavily" (male, 38-58 years, rural).

\section{Availability and accessibility of alcohol}

The majority of participants reported that alcohol is easily available due to the increasing number of alcohol shops (even government outlets) close to residential areas: "There is a mall nearby from where people usually buy alcohol. You come and see around 9-10 p.m., every guy will have a bottle in his hand, get a glass from a juice shop, mix alcohol in the juice and drink" (female, high SES, 38-58 years).

People can easily buy alcohol even on dry days, when the sale of alcohol is not permitted and shops are closed: "Yes, it is available round the year, even at 2 a.m." (female, rural, 18-38 years). If someone wants to buy alcohol after closing hours or on a gazetted holiday (a holiday that is declared by the government as mandatory), he or she can buy it by paying extra money. Participants from the rural areas said that liquor can even be delivered at home for a small extra charge.

The participants said that the regulations on the minimum legal drinking age for buying alcohol and prohibitions related to the sale of alcohol are not implemented properly, which increases young people's access to alcohol: "They [the government] say that no one below 25 years of age can buy alcohol, but you can see children of the age of around 15 years or so buying alcohol. There is no prohibition at all. The government is only interested in earning [revenue]” (male, high SES, 38-58 years).

\section{Reasons for drinking alcohol}

The participants identified peer pressure, "bad" company, curiosity, ideas of alcohol being a fashion or status symbol, enjoyment, getting relief from stress and household tensions, and addiction as some of the reasons for drinking: "The reasons why people drink are because it is in fashion ... a mode of reducing anxiety ... for relaxation, to reduce work pressure, to maintain status” (female, high SES, 3958 years).

Drinking under social pressure was one of the most common reasons for initiating alcohol use. Young people 
usually start consuming alcohol, out of curiosity and to follow the example of individuals in their circle (friends, parents, relatives, etc.): "If the child makes friends with those who drink, the child will start drinking alcohol and it will increase over time" (female, low SES, urban, 18-38 years); "People motivate each other to take one peg [30-90 $\mathrm{ml}$. . . . they say, 'Have it, it will give you energy and zeal'” (male, high SES, 38-58 years).

Some people consider drinking as a source of amusement or enjoyment. For those of high SES, it is a status symbol, a fashion statement, or a way to keep up with the social trends. Most people view drinking as a means of relieving stress after working long hours, alleviating household tensions, easing anxiety, and getting a sound sleep: "People drink alcohol to come out of their sadness and failures in life" (male, low SES, 18-38 years).

Participants of high SES said that some people consume alcohol due to health reasons as well. People believe that drinking alcohol can help to dissolve renal stones and to relieve common cough and cold.

People working in the corporate sector drink to rise in their career: "There are many reasons people start drinking in seniors' company. They feel that they will become the confidante of the person who initiates them into drinking, [for example] their boss. They forget the harm that alcohol will do to them and only think of climbing the career ladder as they have now become a drinking partner of the boss" (female, high SES, 38-58 years).

\section{Discussion}

Alcohol consumption in India has increased substantially over the past few years. This is largely an expected consequence of growing affluence, aggressive marketing, and easy availability of alcohol. Changing social scenarios and weak policies are unable to control the use of alcohol, particularly among children and women. The implementation of laws and policies related to underage drinking and the accessibility of alcohol to minors leaves much to be desired. In most states of India, taxes from alcohol contribute substantially to the state revenue, leading to a conflict of interest between the excise and taxation department and the health department.

Increased availability. Most of the participants observed that the availability of alcohol has increased with the rise in the number of outlets at various locations. These observations on the increase in the production and sale of alcohol are supported by producers and excise departments (Alcoholic Beverages Industry in India, 2013-15). In view of the ban on advertising of alcohol, producers promote their beverages through surrogate advertising and sponsorship. A study conducted in Bangalore, India, reported that nearly two-thirds of participants consumed alcohol in liquor shops, restaurants, bars, and pubs (Girish, Kavita, Gururaj, \& Benegal, 2010).
Weak enforcement of rules and regulations. The participants observed that rules on matters such as dry days, operating hours of shops, and sale of alcohol to underage persons are poorly enforced. Alcohol can be purchased at any time with extra money. Effective policies need to be implemented and best practices for alcohol control put in place (Anderson, Chisholm, \& Fuhr, 2009). Policy gaps need to be addressed through introducing comprehensive alcohol control policies and effective enforcement of strict alcohol control measures (Public Health Foundation of India, 2013). Comprehensive alcohol control policies need to prioritize the public health perspective against the prevailing revenue-generation perspective, as was succinctly put by one participant: "There is no prohibition at all. They are only interested in earning [revenue].”

Increased acceptance of drinking. All participants agreed that alcohol has become a more acceptable part of daily life, contributing to an increase in drinking. This was attributed to aggressive promotion, increased access, and enticing surrogate advertisements for brands by alcohol industry. Rapid urbanization and exposure to western influences were perceived to play an important role, as was the relaxation of overseas trade rules.

Increased drinking among women. Changing social norms have led to an increase in drinking among women, mostly those of high SES and in urban settings, but this is not yet corroborated by the country's survey data.

\section{Conclusions}

This study highlights clear shifts in attitudes to drinking, which is increasingly seen as a part of daily life. The availability of alcohol is perceived to be increasing, and restrictions are not adequately enforced. There are still major differences in attitudes to drinking by males versus females, but alcohol consumption by women of higher SES is becoming a more acceptable norm. These observations strongly suggest that the trend of increasing alcohol consumption in these two northern states is likely to continue and will lead to an escalation of alcohol-related problems. The findings of this study call for an urgent reevaluation of the alcohol policy in India from a public health perspective.

\section{Acknowledgments}

We are thankful to our non-governmental organization partners in the two states where study was undertaken for their support and participation in this study. We are grateful to the former Swedish National Institute of Public Health for collaborating on this study. This research would not have been possible without the participation of community members from selected urban and rural areas of Delhi and Haryana. We also acknowledge the research grant support received from the Swedish International Development Cooperation Agency for this study (Grant number: 2111051). 


\section{References}

Alcoholic Beverages Industry in India. (2013-15). Market Publishers, Report database. Retrieved from: http://www.marketresearch.com/IS-Advisorsv3900/ALCOHOLIC-BEVERAGES-INDIA7429124/

Anderson, P., Chisholm, D., \& Fuhr, D. C. (2009). Effectiveness and cost-effectiveness of policies and programmes to reduce the harm caused by alcohol. The Lancet, 373(9682), 2234-2246. doi:10.1016/S0140-6736(09)60744-3

Benegal, V., Gururaj, G., \& Murthy, P. (2003). Report on a WHO collaborative project on unrecorded consumption of alcohol in Karnataka, India. Retrieved from http://nimhans.ac.in/cam/sites/default/files/ Publications/25.pdf

Bennett, L. A., Campillo, C., Chandrashekar, C. R., \& Gureje, O. (1998). Alcoholic beverage consumption in India, Mexico, and Nigeria: A cross-cultural comparison. Alcohol Health and Research World, 22(4), 243-252.

Esser, M. B., \& Jernigan, D. H. (2015). Multinational alcohol market development and public health: Diageo in India. American Journal of Public Health, 105(11), 2220-2227.

Farooqi, A., Nagra, D., Edgar, T., \& Khunti, K. (2000). Attitudes to lifestyle risk factors for coronary heart disease amongst South Asians in Leicester: A focus group study. Family Practice, 17(4), 293-297.
Girish, N., Kavita, R., Gururaj, G., \& Benegal, V. (2010). Alcohol use and implications for public health: Patterns of use in four communities. Indian Journal of Community Medicine, 35(2), 238-244. doi:10.4103/0970-0218.66875

Gururaj, G., Murthy, P., Rao, G. N., \& Benegal, V. (2011). Alcohol related harm: Implications for public health and policy in India. Bangalore, India: National Institute of Mental Health and Neurosciences.

Maheshwari, B. (2004). Women's anti-alcohol movement in Haryana: Successes and failures. Social Change, 34(1), 40-48.

National Sample Survey Organization. (2005). Household consumer expenditure in India (Report No. 505, 60th Round). New Delhi, India: Ministry of Statistics \& Programme Implementation, Government of India.

Nazar, G., Arora, M., Gupta, V. K., Singh, D., \& Sargent, J. D. (2012). Exposure to alcohol in Bollywood movies and its impact on alcohol use among urban Indian adolescents. Circulation, 125, e703-704.

Public Health Foundation of India. (2013). Alcohol marketing and regulatory policy Environment in India. Retrieved from http://www.phfi.org/images/ events/alcohol_monitioring_report.pdf

Sassi, F. (Ed.). (2015). Tackling harmful alcohol use: Economics and public health policy. Paris, France: OECD Publishing. doi:10.1787/9789264181069-en

World Health Organization. (2011). Global status report on alcohol and health 2011. Geneva, Switzerland: Author. 